\title{
Optimisation of Calcium Alginate and Microbial Transglutaminase Systems to form a Porcine Myofibrillar Protein Gel
}

\author{
Geun Pyo Hong and Koo Bok Chin* \\ Department of Animal Science and Biotechnology Research Institute, Chonnam National University, \\ Gwangju 500-757, Korea
}

\begin{abstract}
The aim of this study was to model and optimize the calcium alginate (CA) and microbial transglutaminase (TG) systems to form a cold-set myofibrillar protein (MP) gel containing $0.1 \mathrm{M}$ or $0.3 \mathrm{M} \mathrm{NaCl}$ using a response surface methodology. The gel strengths of cold-set and heat-induced MP gels, and cooking yields were measured. All measured parameters showed determination coefficients $\left(\mathrm{R}^{2}\right)$ above 0.7 without a lack-of-fit. The CA system had the best results with component ratios of 1.0:0.3:1.0 corresponding to sodium alginate, calcium carbonate and glucono- $\delta$-lactone, respectively, and was favourable at $0.1 \mathrm{M} \mathrm{NaCl}$. In contrast, the TG system only had an effect on cold-set MP gelation at $0.3 \mathrm{M}$ salt, and the optimal ratio of TG to sodium caseinate was $0.6: 0.5$. By combining the two systems at $0.3 \mathrm{M} \mathrm{NaCl}$, an acceptable cold-set $\mathrm{MP}$ gel with an improved texture and high cooking yield could be formed. Therefore, these results indicated that the functionality of the cold-set MP gel could be enhanced by combining these two optimized gelling system.
\end{abstract}

Key words : sodium alginate, transglutaminase, cold-set, myofibrillar, gelation

\section{Introduction}

There have been numerous studies on the various factors affecting cold-set muscle protein gels. It was generally accepted that microbial transglutaminase (TG) and calcium alginate (CA) were the useful cold-set gelling agents (Boles and Shand, 1998; Kuraish et al., 1997). The effects of TG on cold-set myofibrillar protein (MP) gelation showed some contradictions depending on several factors such as potential substrate sources, salt concentration, $\mathrm{pH}$, muscle type, processing time and temperature (Anema et al., 2005; Chin et al., 2009; Kuraish et al., 1997; Kütemeyer et al., 2005). Among potential substrate sources, MPs definitely acted as a substrate for TG, while the quantity of substances contributing to TG activation in MPs, e.g., troponin, were not enough to activate TG (Bergamini et al., 1995). Therefore, sodium caseinate (SC) could be considered as one of the best TG substrates (Kuraish et al., 1997). However, as SC had some negative effects on MPs interactions (Endres and Monagle, 1987; Kilic, 2003), the optimum concentration of SC for TG

*Corresponding author : Koo Bok Chin, Department of Animal Science, Chonnam National University, Gwangju 500-757, Korea. Tel: 82-62-530-2121, Fax: 82-62-530-2129, E-mail: kbchin@chonnam.ac.kr activation should be established.

On the other hand, the application of a calcium alginate (CA) system on cold-set MP gelation had textural problems. As a cold-set gelling system, the CA system consisted of multiple components (i.e., sodium alginate (SA), calcium carbonate $(\mathrm{CC})$ and glucono- $\delta$-lactone $(\mathrm{GdL}))$ and also had a salt-sensible nature (Boles and Shand, 1998). In addition, the CA system lost their gelling nature with the increase in salt concentration, limiting the applications of the CA system on meat product manufacturing (Draget et al., 1991; Ha et al., 2000). However, Hong et al. (2006) suggested that GdL itself was able to form a MP gel, and therefore the CA system could be used at relatively high salt concentrations by optimising the levels of its systematic components. For use of the CA system in meat products, no information on the optimum combination of the components of the CA system was available. Draget et al. (1991) postulated that the optimal amounts of $\mathrm{CC}$ and GdL for $1 \%$ alginate were $0.15 \%$ and $0.5 \%$, respectively. However, high amounts of SA disrupted MP gel texture, thus more detailed information on the optimum component levels of the CA system for MPs were required (Montero et al., 2000).

This study aimed to establish the optimum concentrations of two cold-set MP gelling systems, i.e., TG and CA systems, at $0.1 \mathrm{M}$ and $0.3 \mathrm{M}$ salt concentrations and to 
evaluate the effects of a combined TG and CA system at $0.3 \mathrm{M}$ salt concentration on MP gel properties.

\section{Materials and Methods}

\section{Materials}

Porcine M. longissimus dorsi, with $\mathrm{pH}$ 5.5-5.7, were randomly selected from three carcasses $24 \mathrm{~h}$ post-mortem from a local abattoir. All visible fat and connective tissue were trimmed from the pork meat and cut into $1 \mathrm{~cm}$ cubes. The meat cubes were divided into $200 \mathrm{~g}$ portions, vacuum-packed in a polyethylene pouch, and frozen at $-70^{\circ} \mathrm{C}$ prior to use. Microbial transglutaminase (TG, Activa-TI, $1 \%$ TG with $99 \%$ maltodextrin) was provided by Ajinomoto Food Ingredients (Chicago, IL, USA), and partially hydrolyzed sodium caseinate (SC, HMP 26) was provided by American Casein Company (Burlington, NJ, USA). All other chemicals were analytical grade.

\section{Extraction of Myofibrillar Protein (MP)}

Frozen cubed meat samples were thawed at $4^{\circ} \mathrm{C}$ overnight, and MP was extracted according to a previously published method by Xiong (1993) with minor modifications (Chin et al., 2009). Minced meat was washed three times with 4 vol. (w/v) of $0.1 \mathrm{M} \mathrm{NaCl}, 50 \mathrm{mM}$ sodium phosphate buffer ( $\mathrm{pH}$ 6.25), and then the myofibrillar solution was filtered and adjusted to $\mathrm{pH}$ 6.25. Next, the solution was washed with 8 vol. (w/v) of $0.1 \mathrm{M} \mathrm{NaCl}$ solution ( $\mathrm{pH}$ 6.25). The washed samples were centrifuged at 3,000 $\mathrm{g}$ for $15 \mathrm{~min}$ at $4^{\circ} \mathrm{C}$ using an auto refrigerated centrifuge (J2-21, Beckman Inc, USA) between each washing steps. After extraction of MP, the protein concentration of pellet was determined by Biuret method (Gornall et al., 1949) using bovine serum albumin (Sigma-aldrich, USA) as a standard. All extraction procedures were conducted in a walk-in cooler $\left(4^{\circ} \mathrm{C}\right)$.

\section{Sample Preparation}

The MP sols were formulated as shown in Table 1. In this study, the TG system consisted of TG and SC, and CA systems with SA, CC and GdL. All ingredients were individually diluted in $50 \mathrm{mM}$ sodium phosphate buffer ( $\mathrm{pH}$ 6.25) containing final salt concentrations of either 0.1 or $0.3 \mathrm{M}$. TG, CC and GdL solutions were prepared just prior to mixing all ingredients. For analysis, $5 \mathrm{~g}$ aliquots of samples were loaded into glass tubes ( $\varnothing 12 \mathrm{~mm})$, and incubated at $4^{\circ} \mathrm{C}$ for up to $24 \mathrm{~h}$.

Table 1. Experimental design for modeling the myofibrillar protein gel characteristics

\begin{tabular}{|c|c|c|c|c|c|c|c|c|c|}
\hline \multirow{3}{*}{ Treatments } & \multirow{2}{*}{\multicolumn{3}{|c|}{ Coded unit }} & \multicolumn{6}{|c|}{ Uncoded value } \\
\hline & & & & \multicolumn{3}{|c|}{ CA system $^{1)}$} & \multicolumn{3}{|c|}{ TG system ${ }^{2)}$} \\
\hline & $\mathrm{X}_{1}$ & $\mathrm{X}_{2}$ & $\mathrm{X}_{3}$ & $\mathrm{SA}(\%)$ & $\mathrm{CC}(\%)$ & GdL (\%) & Salt (M) & TG $(\%)$ & $\mathrm{SC}(\%)$ \\
\hline 1 & 0 & 0 & 0 & 0.50 & 0.20 & 0.50 & 0.20 & 0.60 & 0.75 \\
\hline 2 & 0 & 0 & -1.4 & 0.50 & 0.20 & 0.00 & 0.20 & 0.60 & 0.00 \\
\hline 3 & 0 & 0 & 0 & 0.50 & 0.20 & 0.50 & 0.20 & 0.60 & 0.75 \\
\hline 4 & 0 & 1.4 & 0 & 0.50 & 0.40 & 0.50 & 0.20 & 1.20 & 0.75 \\
\hline 5 & 1.4 & 0 & 0 & 1.00 & 0.20 & 0.50 & 0.30 & 0.60 & 0.75 \\
\hline 6 & 1 & 1 & -1 & 0.85 & 0.34 & 0.15 & 0.27 & 1.02 & 0.22 \\
\hline 7 & -1 & 1 & 1 & 0.15 & 0.34 & 0.85 & 0.13 & 1.02 & 1.28 \\
\hline 8 & 1 & -1 & 1 & 0.85 & 0.06 & 0.85 & 0.27 & 0.18 & 1.28 \\
\hline 9 & 0 & 0 & 0 & 0.50 & 0.20 & 0.50 & 0.20 & 0.60 & 0.75 \\
\hline 10 & 0 & 0 & 1.4 & 0.50 & 0.20 & 1.00 & 0.20 & 0.60 & 1.50 \\
\hline 11 & -1 & -1 & 1 & 0.15 & 0.06 & 0.85 & 0.13 & 0.18 & 1.28 \\
\hline 12 & 0 & 0 & 0 & 0.50 & 0.20 & 0.50 & 0.20 & 0.60 & 0.75 \\
\hline 13 & -1.4 & 0 & 0 & 0.00 & 0.20 & 0.50 & 0.10 & 0.60 & 0.75 \\
\hline 14 & 1 & 1 & 1 & 0.85 & 0.34 & 0.85 & 0.27 & 1.02 & 1.28 \\
\hline 15 & -1 & -1 & -1 & 0.15 & 0.06 & 0.15 & 0.13 & 0.18 & 0.22 \\
\hline 16 & 0 & 0 & 0 & 0.50 & 0.20 & 0.50 & 0.20 & 0.60 & 0.75 \\
\hline 17 & 0 & -1.4 & 0 & 0.50 & 0.00 & 0.50 & 0.20 & 0.00 & 0.75 \\
\hline 18 & 0 & 0 & 0 & 0.50 & 0.20 & 0.50 & 0.20 & 0.60 & 0.75 \\
\hline 19 & -1 & 1 & -1 & 0.15 & 0.34 & 0.15 & 0.13 & 1.02 & 0.22 \\
\hline 20 & 1 & -1 & -1 & 0.85 & 0.06 & 0.15 & 0.27 & 0.18 & 0.22 \\
\hline
\end{tabular}

${ }^{1)} \mathrm{CA}$, calcium alginate; SA, sodium alginate; $\mathrm{CC}$, calcium carbonate; GdL, glucono- $\delta$-lactone.

${ }^{2)} \mathrm{TG}$, transglutaminase; $\mathrm{SC}$, sodium caseinate. 


\section{Gel Strength}

Puncture tests were performed to determine the gel strength of MP with cold-setting. The samples $(12 \mathrm{~mm}$ diameter and $20 \mathrm{~mm}$ height) were incubated at $4{ }^{\circ} \mathrm{C}$ for 24 $\mathrm{h}$ and compressed to a breaking point using an Instron universal testing machine (3340, Instron Corporation, Canton, MA, USA) with a stainless steel probe $(9 \mathrm{~mm}$ diameter). Head speed was maintained at $50 \mathrm{~mm} / \mathrm{min}$. Heat-induced gels were heated by increasing the temperature from 0 to $75^{\circ} \mathrm{C}$ at a $3^{\circ} \mathrm{C} / \mathrm{min}$ increment using a thermostat. After heating, samples were cooled in ice and tempered at $4^{\circ} \mathrm{C}$ for $4 \mathrm{~h}$. The analyses were performed in the same method as the cold-set gels.

\section{Cooking Yield}

Before the measurement of puncture test, the exudates in heat-induced gels were discarded. Cooking yields was determined by assessing the weight of gels, and expressed as a percentage of initial weight.

\section{Optimization Process}

All measured graphics were corrected and superimposed. The maximal limitation of dependent variables adopted in the current study was more than 50 gf of coldset MP gel strength, 100 gf of thermal treated cold-set MP gel strength and $85 \%$ of cooking yield using Photoshop CS2 software (ver. 9.0). A superimposed region where all estimated parameters were higher than the limit was assumed as the optimum level for both TG and CA systems.

\section{Statistical Analysis}

Response surface methodology was adopted to designing a mathematical model using SAS 9.1 (SAS Institute, Inc., Cary, NC). The central composite design was employed to study the combined effect of three independent variables coded as $X_{1}, X_{2}$ and $X_{3}$. To simplify the data analysis, SA, CC and GdL levels were adopted as independent variables of SA system, and the experiment was conducted at $0.1 \mathrm{M}$ or $0.3 \mathrm{M}$ salt levels. For optimizing the TG system, salt, TG and SC levels were selected as the independent parameters. In combination, SA system at $0.3 \mathrm{M}$ salt level was repeated in the presence of $0.6 \%$ TG and $0.5 \%$ SC. The complete design consisted of 20 groups having six replications of the center point to estimate pure error. Each parameter was represented by a second-order polynomial expression as:

$$
Y=\beta_{o}+\sum_{i}^{k} \beta_{i} X_{i}+\sum_{i}^{k} \beta_{i i} X_{i}^{2}+\sum_{i<j}^{k} \beta_{i j} X_{i} X_{j}
$$

where $Y$ was the response estimated from replicate measurements, $\beta_{0}, \beta_{\mathrm{i}}, \beta_{\mathrm{ii}}$ and $\beta_{\mathrm{ij}}$ were coefficients from constant, linear, quadratic and interaction terms, respectively. $X_{i}$ and $X_{j}$ were the levels of independent variables, and $k$ was the number of independent variables. The significances of all terms in the polynomial were judged statistically by computing the $F$-value at a probability $(p)$ of $0.001,0.01$ or 0.05 as described by Lee et al. (2006).

\section{Results and Discussion}

\section{Statistical Analysis}

Table 2 shows the experimental responses of each treatment. Regression coefficients of response from each dependent variable are given in Table 3 . The results showed that the generated regression models were able to explain the data variations well and significantly represented the relationship between independent variables and responses. All models were significant $(p<0.001)$ without lack-of-fit and had R-square values greater than 0.7 (Table 3), which showed that the estimated model would be able to explain the results of this study.

\section{Calcium Alginate (CA) System}

In the current study, all data collected from the CA system were sorted by SA and GdL levels at $0.2 \% \mathrm{CC}$ level (Fig. 1). For cold-set MP gel strength, increasing the levels of both SA and GdL proportionally increased the MP gel strength (Fig. 1a, b), with apparently greater effects at $0.1 \mathrm{M}$ than $0.3 \mathrm{M}$ salt. In addition, the gel strength increased with increasing $\mathrm{CC}$ concentration. In the regression model (Table 3), CC showed a significant linear and quadratic effects on cold-set MP gel strength $(p<0.001)$. SA showed a significant quadratic terms $(p<0.05)$, however, GdL alone had no effects on gel strength $(p>0.05)$.

However, all systematic components showed significant interactions with each other on cold-set MP gel strength $(p<0.001)$. These results revealed a reaction sequence of systematic components during cold-set MP gel formation. According to Moneto et al. (2008), SA could not directly interact with MP and form a SA gel network, as the CA system showed no or only limited changes in protein interactions comparing to the control (non-CA system). Calcium ions were the most important factor in SA gel formation; thereby $\mathrm{CC}$ had a highly significant effect on cold-set MP gelation. GdL itself has a potential to form a MP gel (Ngapo et al., 1996), but the GdL effects on MP gelation were not observed at low salt concentration $(0.1 \mathrm{M})$ in this study. Consequently, the 
Table 2. Responses of myofibrillar protein gel characteristics

\begin{tabular}{|c|c|c|c|c|c|c|c|c|c|}
\hline \multirow{3}{*}{ Treatments } & \multicolumn{3}{|c|}{ Calcium alginate system ( $0.1 \mathrm{M}$ salt $)$} & \multicolumn{3}{|c|}{ Calcium alginate system (0.3 M salt) } & \multicolumn{3}{|c|}{ Transglutaminase system } \\
\hline & \multicolumn{2}{|c|}{ Gel strength (gf) } & \multirow{2}{*}{$\begin{array}{l}\text { Yield } \\
(\%)\end{array}$} & \multicolumn{2}{|c|}{ Gel strength (gf) } & \multirow{2}{*}{$\begin{array}{l}\text { Yield } \\
(\%)\end{array}$} & \multicolumn{2}{|c|}{ Gel strength (gf) } & \multirow{2}{*}{ Yield $(\%)$} \\
\hline & Cold-set & Heat-induced & & Cold-set & Heat-induced & & Cold-set & Heat-induced & \\
\hline 1 & 124.0 & 117.8 & 93 & 24.3 & 26.1 & 100 & 13.0 & 50.5 & 54 \\
\hline 2 & 11.7 & 9.8 & 100 & 13.3 & 42.1 & 100 & 11.3 & 62.2 & 61 \\
\hline 3 & 124.5 & 117.1 & 92 & 23.7 & 28.0 & 100 & 12.2 & 43.9 & 54 \\
\hline 4 & 127.4 & 115.8 & 94 & 28.4 & 30.9 & 100 & 14.3 & 31.5 & 55 \\
\hline 5 & 196.1 & 195.6 & 99 & 54.8 & 34.7 & 100 & 82.0 & 142.5 & 100 \\
\hline 6 & 73.6 & 25.5 & 100 & 31.2 & 23.5 & 100 & 42.8 & 89.6 & 67 \\
\hline 7 & 28.7 & 28.0 & 67 & 13.8 & 31.5 & 89 & 7.6 & 26.2 & 53 \\
\hline 8 & 167.9 & 152.9 & 95 & 39.8 & 39.1 & 100 & 10.0 & 43.0 & 71 \\
\hline 9 & 119.1 & 116.9 & 90 & 23.4 & 26.2 & 100 & 11.3 & 40.7 & 53 \\
\hline 10 & 198.0 & 233.2 & 84 & 35.1 & 43.9 & 87 & 11.6 & 57.2 & 54 \\
\hline 11 & 16.0 & 35.2 & 71 & 10.2 & 33.8 & 91 & 3.8 & 30.3 & 56 \\
\hline 12 & 123.6 & 118.4 & 94 & 22.5 & 23.6 & 100 & 13.6 & 54.7 & 55 \\
\hline 13 & 3.1 & 24.3 & 60 & 11.8 & 28.9 & 88 & 5.7 & 33.1 & 54 \\
\hline 14 & 335.1 & 375.4 & 93 & 72.5 & 64.5 & 92 & 36.7 & 79.3 & 61 \\
\hline 15 & 3.9 & 5.0 & 100 & 9.5 & 25.5 & 100 & 8.2 & 49.8 & 54 \\
\hline 16 & 121.5 & 111.8 & 88 & 23.8 & 24.1 & 100 & 12.0 & 53.8 & 55 \\
\hline 17 & 2.8 & 11.8 & 86 & 5.3 & 13.8 & 96 & 2.7 & 14.4 & 88 \\
\hline 18 & 116.7 & 121.9 & 92 & 23.3 & 22.5 & 100 & 13.4 & 47.6 & 51 \\
\hline 19 & 8.2 & 5.4 & 98 & 12.3 & 31.4 & 100 & 14.6 & 40.6 & 55 \\
\hline 20 & 23.9 & 6.0 & 100 & 14.7 & 15.3 & 100 & 18.1 & 46.8 & 72 \\
\hline
\end{tabular}

Table 3. Regression coefficients of models fitted from central composite design

\begin{tabular}{|c|c|c|c|c|c|c|c|c|c|}
\hline \multirow{3}{*}{ Terms } & \multicolumn{3}{|c|}{ 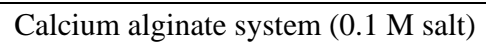 } & \multicolumn{3}{|c|}{ Calcium alginate system ( $0.3 \mathrm{M}$ salt $)$} & \multicolumn{3}{|c|}{ Transglutaminase system } \\
\hline & \multicolumn{2}{|c|}{ Gel strength } & \multirow{2}{*}{ Yield } & \multicolumn{2}{|c|}{ Gel strength } & \multirow{2}{*}{ Yield } & \multicolumn{2}{|c|}{ Gel strength } & \multirow{2}{*}{ Yield } \\
\hline & Cold-set & Heat-induced & & Cold-set & Heat-induced & & Cold-set & Heat-induced & \\
\hline$\beta_{0}$ & -12.3 & 33.2 & 97.9 & 15.9 & 46.2 & 93.4 & 69.4 & 142.0 & 81.7 \\
\hline \multicolumn{10}{|l|}{ Linear } \\
\hline$\beta_{1}$ & -26.2 & $-121.1 *$ & $33.7 * *$ & $-52.2 * * *$ & $-55.9 * * *$ & $18.6^{* *}$ & $-894.0 * * *$ & $-1271.2 * * *$ & -346.8 \\
\hline$\beta_{2}$ & $348.5^{* * * *}$ & $265.8^{*}$ & -16.9 & $43.6 * *$ & 32.9 & 14.4 & 14.8 & 48.9 & $-24.2 *$ \\
\hline$\beta_{3}$ & -37.3 & $-108.4 *$ & $-59.8 * * *$ & $-20.1 * * *$ & $-79.0 * * *$ & 4.8 & 17.3 & -33.3 & 21.3 \\
\hline \multicolumn{10}{|l|}{ Quadratic } \\
\hline$\beta_{11}$ & $-67.8^{*}$ & -53.5 & $-34.5 * * *$ & $39.4 * * *$ & $19.5^{*}$ & $-14.8 * *$ & $2529.3 * * *$ & $3263.4 * * *$ & $1339.8 * *$ \\
\hline$\beta_{22}$ & $-1285.4 * * *$ & $-1489.4 * * *$ & 47.1 & $-166.0 * * *$ & -113.3 & 7.7 & $-28.1^{*}$ & $-89.5 * * *$ & 21.9 \\
\hline$\beta_{33}$ & -46.7 & -7.3 & 15.5 & 3.0 & $64.3 * * *$ & $-16.8 * * *$ & -12.7 & 8.0 & -10.8 \\
\hline \multicolumn{10}{|l|}{ Interaction } \\
\hline$\beta_{12}$ & $500.1 * * *$ & $621.8 * * *$ & 10.0 & $107.1 * * *$ & $74.8^{* *}$ & -15.0 & $172.2 *$ & $384.4 * *$ & -54.2 \\
\hline$\beta_{13}$ & $372.9 * * *$ & $444.0 * * *$ & $48.0 * * *$ & $64.2 * * *$ & $56.2 * * *$ & $12.0 *$ & -9.8 & 65.9 & -23.3 \\
\hline$\beta_{23}$ & $314.7 * * *$ & $486.6^{* * *}$ & -10.0 & $42.7 * * *$ & 22.6 & $-25.0 *$ & -0.3 & -0.8 & -5.0 \\
\hline $\mathrm{R}^{2}$ & 0.98 & 0.96 & 0.87 & 0.99 & 0.86 & 0.82 & 0.83 & 0.82 & 0.70 \\
\hline
\end{tabular}

$* p<0.05, * * p<0.01, p<0.001$.

effect of GdL on cold-set MP gelation must be dependent on the levels of SA and CC, which led to the lack of GdL significance in the regression model.

The gel strength of heat-induced gel was similar to the cold-set with minor changes (Fig. 1c, d). Generally, SA alone might interfere in forming the MP gel network during thermal treatment and possibly led to detrimental effects on gel texture (Kilic, 2003; Montero et al., 2000). However, calcium-induced SA gels had an irreversible thermal nature which caused no or minor changes in gel texture after thermal treatment (Renn, 1984). According to the regression model, all components in the SA system had a significant linear $(p<0.05)$ and interaction $(p<$ $0.001)$ effects, and $\mathrm{CC}$ had a significant quadratic term 

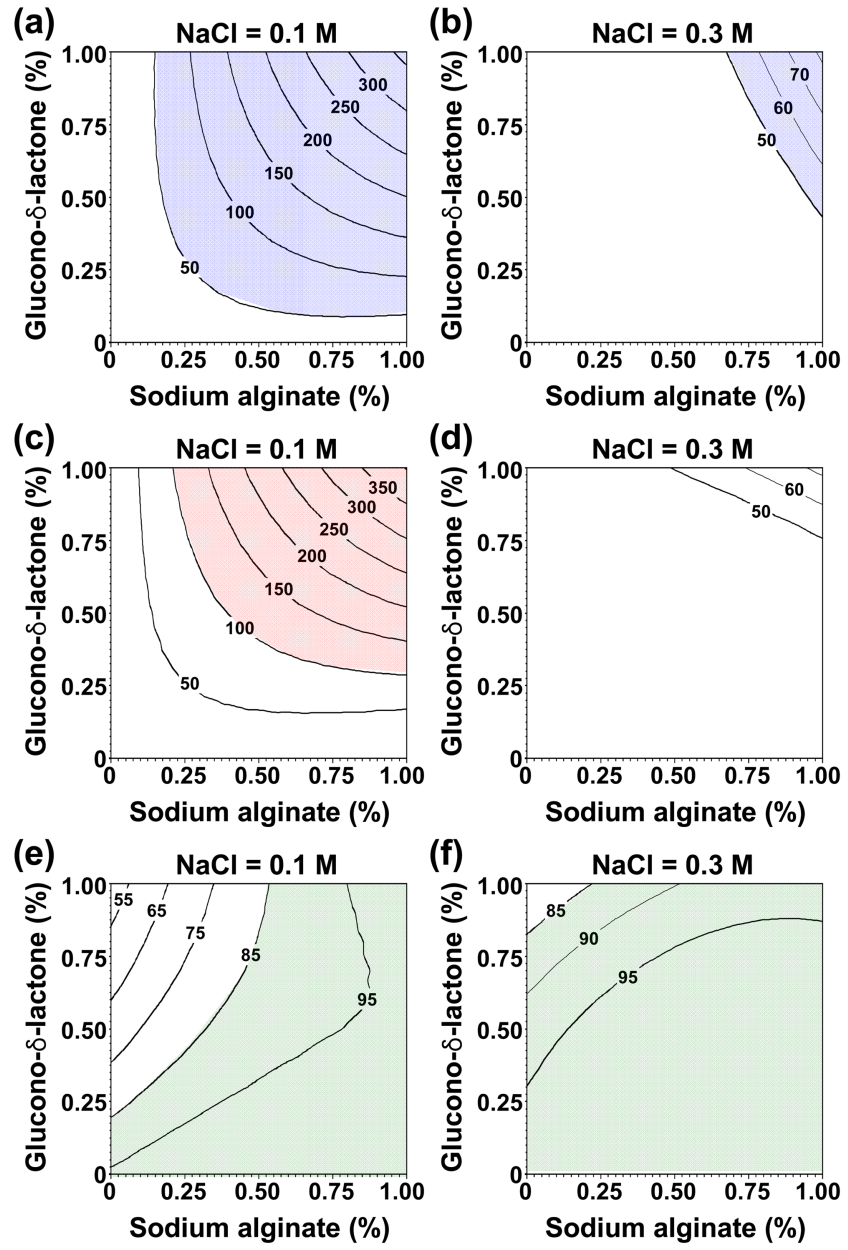

Fig. 1. Contour plots for the calcium alginate system mediated (a, b) cold-set, (c, d) heat-induced gel strengths (gf) and (e, f) cooking yield (\%) of myofibrillar protein at $0.1 \mathrm{M}$ and $0.3 \mathrm{M}$ salt.

$(p<0.001)$ at $0.1 \mathrm{M}$ salt concentration. However, CC lost their gelling capacity at a $0.3 \mathrm{M}$ salt concentration. SA and GdL were the important factors in gel strength of heat-induced MP at $0.3 \mathrm{M}$ salt concentration $(p<0.001)$. The influences of salt concentrations were possibly due to the competition between sodium and calcium ions on the SA binding site (Draget et al., 1991).

For cooking yield (Fig. 1e, f), SA had a significance on linear $(p<0.01)$ and quadratic functions $(p<0.001)$. On the other hand, the effect of GdL on cooking yield varied depending on the salt concentrations; GdL had a linear effect $(p<0.001)$ at $0.1 \mathrm{M}$ salt concentration. However a quadratic effect at $0.3 \mathrm{M}$ salt concentration $(p<0.001)$. However, CC had no effects on cooking yield of MP $(p>0.05)$, indicating that $\mathrm{CC}$ was used only as a calcium source in altering gel characteristics. In this study, the addition of $0.5 \%$ SA showed an cooking yield higher than $85 \%$ at $0.1 \mathrm{M}$ salt, even if high amounts of GdL (>1\%) was added.

Based on these results, the CA system was the best cold-set MP gelling system at low salt levels $(0.1 \mathrm{M})$ and was able to control the gel strength and cooking yield of cold-set MP gels. Cold-set MP gels containing the SA system also maintained their gel strength after thermal treatments. Furthermore, cold-set MP gelation could be achieved at relatively high salt concentration $(0.3 \mathrm{M})$ by proportionally increasing the concentrations of all components in the CA system, although the texture was not improved over the heat-induced gel.

\section{Transglutaminase (TG) System}

Cold-set MP gel without TG was not formed at $0.1 \mathrm{M}$ $\mathrm{NaCl}$, indicating that the TG system could not be effective at low salt levels (Fig. 2a). With $0.3 \mathrm{M}$ salt level, increasing the TG level increased the gel strength of MP, and the maximal increment was found when $0.5 \% \mathrm{SC}$
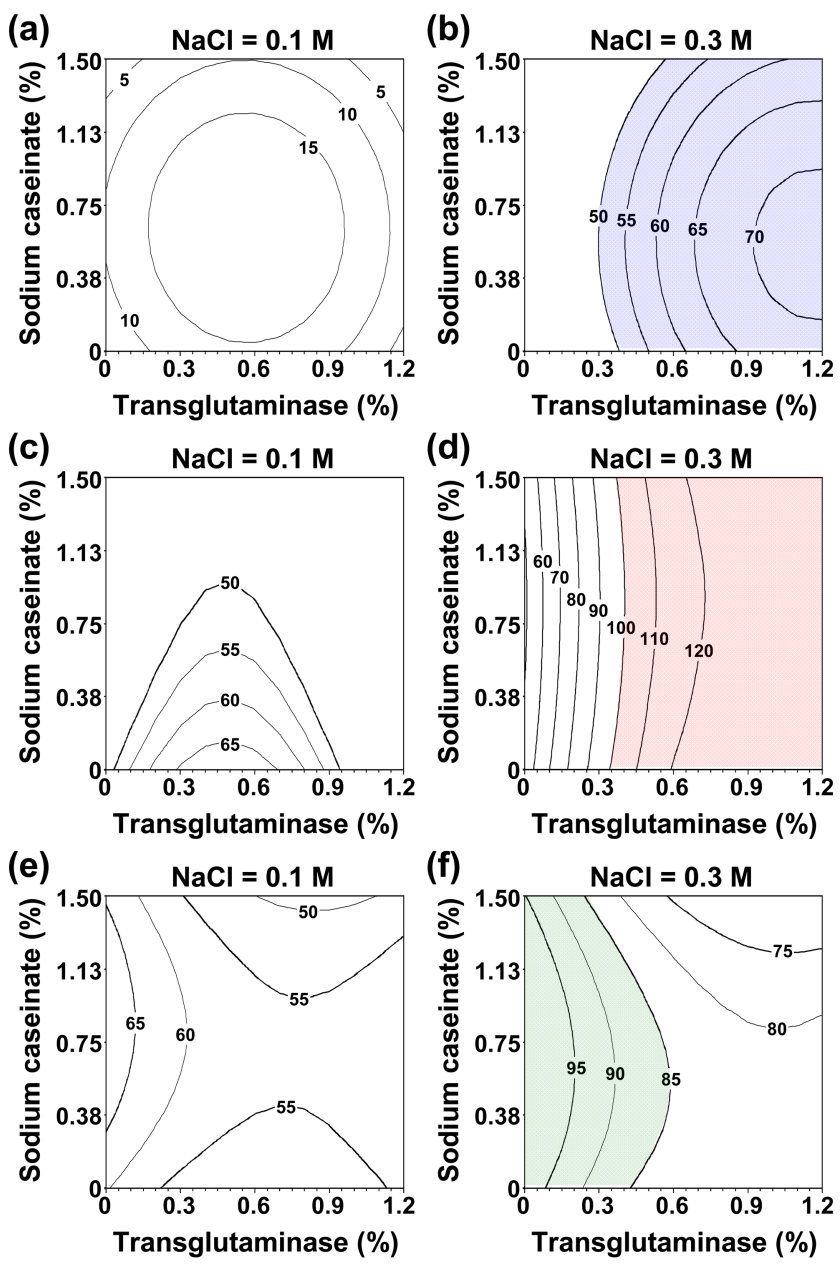

Fig. 2. Contour plots for the microbial transglutaminase system mediated (a, b) cold-set, (c, d) heat-induced gel strengths (gf) and (e, f) cooking yield (\%) of myofibrillar protein at $0.1 \mathrm{M}$ and $0.3 \mathrm{M}$ salt. 
was concurrently added with TG (Fig. 2b). As shown in the model (Table 3), SC had no significant effects on the gel strength of cold-set MP gels $(p>0.05)$. Although TG had a significance in quadratic term $(p<0.05)$, the main contributor in cold-set MP gel formation was salt $(p<0.001)$. The result was provably due to the salt-dependency of TG (Kütemeyer et al., 2005). Kuraish et al. (1997) reported that SC was the best substrate for TG activation at refrigeration temperatures. However, the optimum SC concentration corresponding to a given amount of TG was not clear because various potential factors in activating TG were also present, including $\mathrm{pH}$, salt and protein solubility (Kütemeyer et al., 2005; Nielsen et al., 1995). In this study, optimum SC concentration for cold-set MP gelation was evaluated to be approximately $0.5 \%$, regardless of TG or salt concentrations. However, SC had a negative effect on protein-protein interactions in MPs (Endres and Monagle, 1987), and thus, SC reduced the MP gel strength with TG activation. In addition, the TG used in this study contained maltodextrin (99\%) which is added as a gel softener or fat replacer (Crehan et al., 2000). Increasing TG caused a proportional increase in maltodextrin concentration, since TG used in this study consisted of $1 \%$ pure TG and $99 \%$ maltodextrin. When the salt concentration increased, MP solubility increased, which resulted in high activation of TG with increasing TG concentration.

After heat treatment of MP gels, the gel strength was greatly increased compared to cold-set gels due to the heat activation of TG. However, all estimations of heatinduced MP gel strength were less than $60 \mathrm{gf}$ at $0.1 \mathrm{M}$ salt. According to the regression model, all factors contributing to the gel strength of MP after heating was similar to those of cold-set ones with only slight changes in significance levels. The usage of TG had a drawback as a cold-set gelling system in terms of food hygiene, because of their high activation temperature (Nielsen et al., 1995). However, optimal conditions for TG activation depended on pH and substrate sources (Sakamoto et al., 1994), and Kuraish et al. (1997) reported that TG combined with SC could be activated at refrigerated temperature. In addition, the high activation temperature of TG led to the increase in the gel strength of MP after thermal treatment.

TG was detrimental to the MP gel cooking yield at low salt concentrations (Fig. 2e, f). At $0.3 \mathrm{M}$ salt, increasing TG also decreased the cooking yield. In the regression analysis, TG had a linear effect $(p<0.05)$, and salt concentration showed a significant quadratic term $(p<0.001)$. However, no significant effect was found due to various
SC concentrations. According to the results, the TG system was useful as a cold-set MP gelling system at $0.3 \mathrm{M}$ salt level, and the MP gel texture after heat treatment was highly enhanced by adding the TG system. However, the TG system could be applicable to meat products containing at least $0.3 \mathrm{M}$ salt, but the enhanced protein-protein interactions by TG resulted in low product yield. These results revealed that the combination of CA and TG systems was required to reduce the negative impacts contained in each system.

\section{Optimization}

The optimised combinations of CA and TG systems are given in Fig. 3. Because the excessive addition of the CA system extremely changed the textural properties of the gel (Park, 2000) and the CA system was more effective at lower salt concentrations, the CA system were adopted as the minimal concentrations at which all dependent variables, i.e., gel strengths and yield, showed higher than the targeted values. All combination in the SA system showed the best effects on gel strength and yields at $0.1 \mathrm{M}$ salt, when $0.5 \% \mathrm{SA}, 0.15 \% \mathrm{CC}$ and $0.5 \% \mathrm{GdL}$ were combined. At $0.3 \mathrm{M}$ salt level, the gel strength of the heatinduced MP gel was shown to be less than $100 \mathrm{gf}$, which made it impossible to optimise the MP gel characteristics.

For TG system at $0.1 \mathrm{M}$ salt, there were no optimum levels of dependent variables. Consequently, TG had no effects on cold-set MP gelation at $0.1 \mathrm{M}$ salt concentration. Since the TG system has a salt-dependent nature, the maximal concentrations of TG systems in the superimposed region were established as the best combination for the TG system. Therefore, the optimal component percentages of the TG system were found to be $0.6 \% \mathrm{TG}$ and $0.5 \% \mathrm{SC}$ at $0.3 \mathrm{M}$ salt concentration. In addition, these optimized percentages led to the highest values of depen-
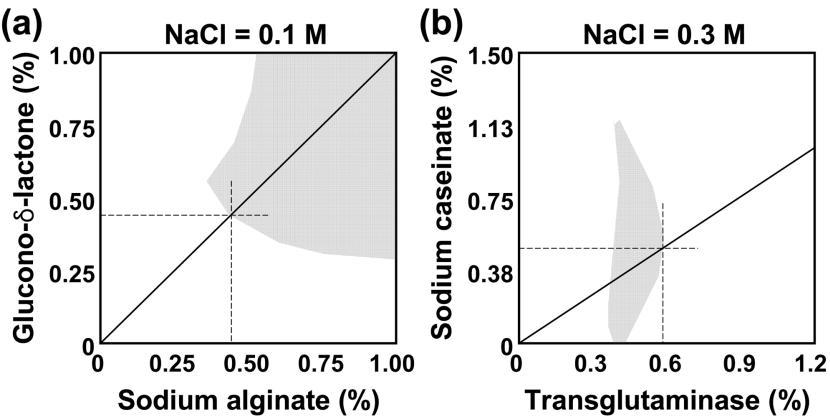

Fig. 3. Optimal regions of (a) the calcium alginate system at $0.1 \mathrm{M}$ salt concentration and (b) the microbial transglutaminase system at $0.3 \mathrm{M}$ salt concentration. Gray dimensions indicated the optimized regions for each system. 
dent variables even at $0.1 \mathrm{M}$ salt concentration.

\section{Combination of TG and CA Systems}

Salt concentration of meat products generally ranged from 1.5 to $2.0 \%$ (about $0.3 \mathrm{M}$ ). Thus, the combination effects of TG and CA systems were established at $0.3 \mathrm{M}$ salt concentration (Fig. 4). Table 4 presents the experimental responses of MP gel characteristics containing $0.6 \% \mathrm{TG}$ and $0.5 \% \mathrm{SC}$ at $0.3 \mathrm{M} \mathrm{NaCl}$. It was expected that TG was contributed to only textural properties of MP gel at $0.3 \mathrm{M}$ salt, and thus, an acceptable MP gel with high moisture might be obtained by combining with the CA system. For cold-set MP gel strength, the CA and TG combination showed the best cold-set MP gel texture (Fig. 4a). The only unacceptable cold-set MP gel strength $(<50$ gf) was shown when a low SA $(<0.4 \%)$ with high GdL $(>0.95 \%)$ ratio were incorporated into the MP gel, probably due to the inactivation of TG under the low $\mathrm{pH}$. However, SA seemed to interfere with TG mediated protein-protein interactions after thermal treatment (Fig. 4b), and thus, the usage of high amount of SA with TG system should be avoided. The cooking yield of MP gel was directly proportional to SA concentration (Fig. 4c). According to the regression model (Table 5), SA and CC had significances in linear and quadratic terms. GdL (a)

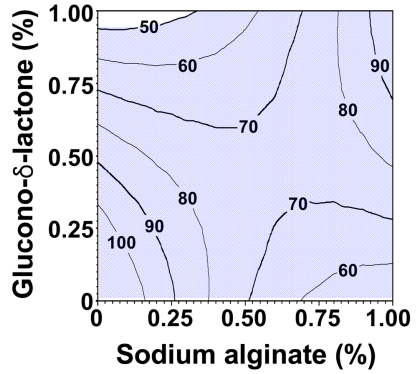

(c)

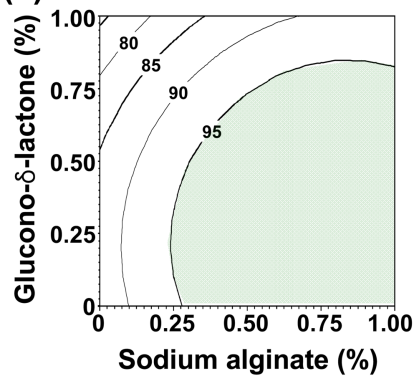

(d)
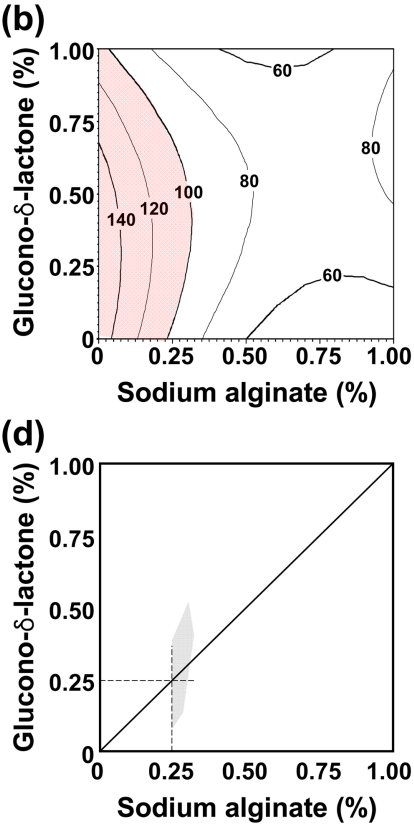

Fig. 4. Contour plots for (a) cold-set, (b) heat-induced gel strengths (gf) and (c) cooking yield (\%) of myofibrillar protein containing $0.6 \%$ transglutaminase and $0.5 \%$ sodium caseinate at a $0.3 \mathrm{M}$ salt concentration, and (d) optimal levels of the calcium alginate system.

showed a linear effect on cold-set MP gel strength $(p<0.05)$, while quadratic effects on heat-induced gel

Table 4. Experimental design and responses of myofibrillar protein gel characteristics containing $0.6 \%$ transglutaminase

\begin{tabular}{|c|c|c|c|c|c|c|c|c|c|}
\hline \multirow{3}{*}{ Treatments } & \multicolumn{6}{|c|}{ Independent variables } & \multicolumn{3}{|c|}{ Dependent variables } \\
\hline & \multicolumn{3}{|c|}{ Coded unit } & \multicolumn{3}{|c|}{ Uncoded value $^{1)}$} & \multicolumn{2}{|c|}{ Gel strength (gf) } & \multirow{2}{*}{ Yield (\%) } \\
\hline & $\mathrm{X}_{1}$ & $\mathrm{X}_{2}$ & $\mathrm{X}_{3}$ & SA $(\%)$ & $\mathrm{CC}(\%)$ & GdL (\%) & Cold-set & Heat-induced & \\
\hline 1 & 0 & 0 & 0 & 0.50 & 0.20 & 0.50 & 86.9 & 89.5 & 100 \\
\hline 2 & 0 & 0 & -1.4 & 0.50 & 0.20 & 0.00 & 63.1 & 68.1 & 100 \\
\hline 3 & 0 & 0 & 0 & 0.50 & 0.20 & 0.50 & 67.7 & 72.0 & 98 \\
\hline 4 & 0 & 1.4 & 0 & 0.50 & 0.40 & 0.50 & 55.7 & 61.9 & 92 \\
\hline 5 & 1.4 & 0 & 0 & 1.00 & 0.20 & 0.50 & 75.3 & 69.5 & 100 \\
\hline 6 & 1 & 1 & -1 & 0.85 & 0.34 & 0.15 & 48.2 & 28.9 & 100 \\
\hline 7 & -1 & 1 & 1 & 0.15 & 0.34 & 0.85 & 56.3 & 88.7 & 91 \\
\hline 8 & 1 & -1 & 1 & 0.85 & 0.06 & 0.85 & 59.7 & 58.1 & 100 \\
\hline 9 & 0 & 0 & 0 & 0.50 & 0.20 & 0.50 & 78.1 & 86.5 & 100 \\
\hline 10 & 0 & 0 & 1.4 & 0.50 & 0.20 & 1.00 & 54.2 & 62.8 & 84 \\
\hline 11 & -1 & -1 & 1 & 0.15 & 0.06 & 0.85 & 44.3 & 62.6 & 77 \\
\hline 12 & 0 & 0 & 0 & 0.50 & 0.20 & 0.50 & 83.4 & 84.8 & 99 \\
\hline 13 & -1.4 & 0 & 0 & 0.00 & 0.20 & 0.50 & 84.2 & 178.1 & 83 \\
\hline 14 & 1 & 1 & 1 & 0.85 & 0.34 & 0.85 & 94.4 & 63.3 & 89 \\
\hline 15 & -1 & -1 & -1 & 0.15 & 0.06 & 0.15 & 82.4 & 90.1 & 86 \\
\hline 16 & 0 & 0 & 0 & 0.50 & 0.20 & 0.50 & 52.6 & 59.4 & 100 \\
\hline 17 & 0 & -1.4 & 0 & 0.50 & 0.00 & 0.50 & 44.2 & 59.1 & 96 \\
\hline 18 & 0 & 0 & 0 & 0.50 & 0.20 & 0.50 & 78.8 & 80.6 & 100 \\
\hline 19 & -1 & 1 & -1 & 0.15 & 0.34 & 0.15 & 101.2 & 107.1 & 96 \\
\hline 20 & 1 & -1 & -1 & 0.85 & 0.06 & 0.15 & 66.8 & 56.6 & 100 \\
\hline
\end{tabular}

1) SA, sodium alginate; CC, calcium carbonate; GdL, glucono- $\delta$-lactone. 
Table 5. Regression coefficients for three models of transglutaminase calcium alginate system

\begin{tabular}{clll}
\hline \hline \multirow{2}{*}{ Terms } & \multicolumn{2}{c}{ Gel strength } & \multirow{2}{*}{ Yield } \\
\cline { 2 - 3 } & \multicolumn{1}{c}{ Cold-set } & Heat-induced & \\
\hline$\beta_{0}$ & 102.4 & 113.3 & 72.0 \\
Linear & & & \\
$\beta_{1}$ & $-114.6^{* * *}$ & $-217.9^{* * *}$ & $54.0^{* * *}$ \\
$\beta_{2}$ & $159.7^{*}$ & $326.5^{* *}$ & $89.7^{* * *}$ \\
$\beta_{3}$ & $-67.4^{*}$ & 27.3 & 11.9 \\
Quadratic & & & \\
$\beta_{11}$ & $54.3^{*}$ & $140.2^{* * *}$ & $-23.1^{* * *}$ \\
$\beta_{22}$ & $-405.3^{* *}$ & $-706.3^{* * *}$ & $-82.1^{*}$ \\
$\beta_{33}$ & -30.1 & $-93.3^{* *}$ & $-21.1^{* * *}$ \\
Interaction & & & \\
$\beta_{12}$ & -36.7 & $-163.8^{*}$ & $-87.5^{* * * *}$ \\
$\beta_{13}$ & $122.1^{* * *}$ & $81.9^{*}$ & 3.0 \\
$\beta_{23}$ & $115.9^{*}$ & 104.8 & -17.5 \\
$\mathrm{R}^{2}$ & 0.69 & 0.81 & 0.88 \\
\hline
\end{tabular}

${ }^{*} p<0.05,{ }^{* *} p<0.01, p<0.001$.

strength $(p<0.01)$ and cooking yield $(p<0.001)$. In the current study, less than $0.3 \%$ of the CA system improved the MP gel yield up to $95 \%$. In conclusion, the optimum combination of the CA system at $0.3 \mathrm{M}$ salt and $0.6 \% \mathrm{TG}$ system was estimated at $0.25 \% \mathrm{SA}$ with $0.08 \% \mathrm{CC}$ and $0.25 \% \mathrm{GdL}$. However, the influence of the TG system at a $0.5 \%$ CA system under low salt concentrations could not be expected because TG itself did not contribute to the MP gel texture at low salt concentrations (Fig. 2a). These results evidently showed that by optimizing the combination of TG and CA systems, acceptable cold-set MP gels could be formed with low salt meat products.

\section{Acknowledgements}

This study was partially supported by the Brain Korea 21 Project from Ministry of Education and Human Resources Development, and by Biotechnology Research Institute, Chonnam National University, Gwangju, Republic of Korea.

\section{References}

1. Anema, S. G., Lauber, S., Lee, S. K., Henle, T., and Klostermeyer, H. (2005) Rheological properties of acid gels prepared from pressure- and transglutaminase-treated skim milk. Food Hydrocolloid 19, 879-887.

2. Bergamini, C. M., Signorini, M., Barbato, R., Menabo, R., Lisa, F. D., Gorza, L., and Beninati, S. (1995) Transglutaminase-catalyzed polymerization of troponin in vitro. Biochem. Biophys. Res. Commun. 206, 201-206.
3. Boles, J. A. and Shand, P. J. (1998) Effect of comminution method and raw binding system in restructured beef. Meat Sci. 49, 297-307.

4. Chin, K. B., Go, M. Y., and Xiong, Y. L. (2009) Konjac flour improved textural and water retention properties of transglutaminase-mediated, heat-induced porcine myofibrillar protein gel: Effect of salt level and transglutaminase incubation. Meat Sci. 81, 565-572.

5. Crehan, C. M., Hughes, E., Troy, D. J., and Buckley, D. J. (2000) Effects of fat level and maltodextrin on the functional properties of frankfurters formulated with 5, 12 and $30 \%$ fat. Meat Sci. 55, 463-469.

6. Draget, K. I., Steinsvåg, K., Onsøyen, E., and Smidsrød, O. (1991) Na- and K-alginate; effect on $\mathrm{Ca}^{2+}$-gelation. Carbohydr. Polym. 35, 1-6.

7. Endres, J. G. and Monagle, C. W. (1987) Nonmeat protein additives. In Advances in meat research. (vol. 3) (pp. 331350) Pearson, A. M. and Cutson, T. R. (eds.) Van Nostrand Reinhold Company: New York.

8. Gornall, A. G., Bardawill, C. Y., and David, M. M. (1949) Determination of serum proteins by means of the biuret reaction. J. Biol. Chem. 177, 751-756.

9. Ha, J. U., Woo, D. K., and Hwang, Y. M. (2000) Effect of carboxy methyl cellulose and methyl cellulose on the functional properties of pork heart alginate/calcium carbonate (AC) surimi. Korean J. Food Sci. Ani. Resour. 20, 199-206.

10. Hong, G. P., Park, S. H., Kim, J. Y., Ko, S. H., and Min, S. G. (2006) Effects of salt, glucono- $\delta$-lactone and high pressure treatment on physico-chemical properties of restructured pork. Korean J. Food Sci. Ani. Resour. 26, 204-211.

11. Kilic, B. (2003) Effect of microbial transglutaminase and sodium caseinate on quality of chicken döner kebab, Meat Sci. 63, 417-421.

12. Kuraishi, C., Sakamoto, J., Yamazaki, K., Susa, Y., Kuhara, C., and Soeda, T. (1997) Production of restructured meat using microbial transglutaminase without salt or cooking. $J$. Food Sci. 62, 488-515.

13. Kütemeyer, C., Froeck, M., Werlein, H. D., and Watkinson, B. M. (2005) The influence of salts and temperature on enzymatic activity of microbial transglutaminase. Food Control. 16, 735-737.

14. Lee, W. C., Yusof, S., Hamid, N. S. A., and Baharin, B. S. (2006) Optimizing conditions for enzymatic clarification of banana juice using response surface methodology (RSM). $J$. Food Eng. 73, 55-63.

15. Monero, H. M., Carballo, J., and Borderías, A. J. (2008) Influence of alginate and microbial transglutaminase as binding ingredients on restructured fish muscle processed at low temperature. J. Sci. Food Agric. 88, 1529-1536.

16. Montero, P., Hurtado, J. L., and Pérez-Mateos, M. (2000) Microstructural behaviour and gelling characteristics of myosystem protein gels interacting with hydrocolloids. Food Hydrocolloid 14, 455-461.

17. Ngapo, T. M., Wilkinson, B. H. P., and Chong, R. (1996) 1,5Glucono- $\delta$-lactone-induced gelation of myofibrillar protein at chilled temperature. Meat Sci. 42, 3-13. 
18. Nielsen, G. S., Petersen, B. R., and Møller, A. J. (1995) Impact of salt, phosphate and temperature on the effect of a transglutaminase (F XIIIa) on the texture of restructured meat. Meat Sci. 41, 293-299

19. Park, J. W. (2000) Surimi and surimi seafood. Marcel Dekker: New York.

20. Renn, D. W. (1984) Marine algae and their role in biotechnology In Biotechnology in the marine sciences: proceedings of the first annual MIT sea grant lecture and seminar (pp. 191-206.) Sinskey, A. J., Pariser, E. R., and Colwell, R. R. (eds.), Willey: New York.

21. Sakamoto, H., Kumazawa, Y., and Motoki, M. (1994) Strength of protein gels prepared with microbial transglutaminase as related to reaction conditions. J. Food Sci. 59, 866-871.

22. Xiong, Y. L. (1993) A comparison of the rheological characteristics of different fractions of chicken myofibrillar proteins. J. Food Biochem. 16, 217-227.

(Received 2009.7.28/Revised 2009.10.5/Accepted 2009.10.9) 